\title{
On the rigidity of rank gradient in a group of intermediate growth
}

\author{
Rostislav Grigorchuk* \\ Texas A\&M University, \\ Rostyslav Kravchenko \\ Northwestern University
}

September 12, 2018

Dedicated to A.M. Samoilenko on the occasion of his 80th birthday

\begin{abstract}
We introduce and investigate the rigidity property of rank gradient in the case of the group $\mathcal{G}$ of intermediate growth constructed by the first author in 10. We show that $\mathcal{G}$ is normally $(f, g)$-RG rigid where $f(n)=\log (n)$ and $g(n)=\log (\log (n))$.
\end{abstract}

\section{Introduction}

A group is said to be residually finite if it has sufficiently many subgroups of finite index so that the intersection of them is trivial. This is important class of groups studied throughout more than eight decades by various tools and means. Residually finite groups are the reach source of examples in group theory. In particular they are often used in the three main branches of modern group theory: geometric group theory, asymptotic group theory and measured group theory. Such groups have realization by actions on spherically homogeneous rooted trees as indicated in [6, 8, which, in many cases, gives a possibility to study them and their subgroup structure using the structure of the tree. They are also closely connected to the theory of profinite groups.

A very important invariant of residually finite group is a subgroup growth introduced by F. Grunewald, D. Segal and G. Smith 11 and studied by many researches (see a comprehensive book 13 and the literature therein on this subject). Recently another asymptotic characteristics of residually finite groups were introduced with the focus on the notion of rank gradient.

\footnotetext{
*The author was partially supported by Simons Collaboration Grant \# 527814

${ }^{\dagger}$ To appear in Ukrainian Mathematical Journal v.70, no. 2 (2018)
} 
The rank gradient of a finitely generated residually finite group $G$ is defined as

$$
R G(G)=\inf \frac{d(H)-1}{[G: H]}
$$

where the infimum is taken over all subgroups $H$ in $G$ of finite index and $d(H)$ is the rank of $H$ (i.e. the minimal number of generators of $H)$. It is a finite number because a subgroup of finite index in a finitely generated group is finitely generated, and the first question that arises is whether $R G(G)=0$ or not.

This notion, as well as the notion of the rank gradient relative to the descending chain of subgroups (defined by (2) ), were introduced for the first time by M. Lackenby [12] with motivation from 3-dimensional topology. Since that the rank gradient and its variations were intensively studied 1, 4, 7, 8, 9, 12.

The definition (10) can be modified in various directions. Instead of inf one can consider sup, instead of all subgroups one can consider only normal subgroups, or subgroups with index a power of a prime number $p$, etc. Another direction of modifications is to consider descending sequence $H_{n}, n=1,2, \ldots$ of subgroups of finite index and associated sequence $r g(n)$ of numbers defined as

$$
r g(n)=R G\left(G,\left\{H_{n}\right\}\right)=\frac{d\left(H_{n}\right)-1}{\left[G: H_{n}\right]}
$$

and its limit

$$
\lim _{n \rightarrow \infty} r g(n)
$$

if it exists, or the upper and the lower limits

$$
\begin{aligned}
& \limsup _{n \rightarrow \infty} r g(n), \\
& \liminf _{n \rightarrow \infty} r g(n)
\end{aligned}
$$

otherwise.

It is known 12, 1, that if $G$ is amenable (the notion of amenable group was introduced by von Neumann [14] and by Bogolyubov in topological case $\left[3\right.$ ), and $\left\{H_{n}\right\}$ is a sequence of subgroups of finite index satisfying some technical condition (sometimes called the Farber condition, it is equivalent to the essential freeness of the action of the group on the boundary of coset tree (7]), then the limit (2) exists and is equal to 0 . The example of the lamplighter group $\mathcal{L}=\mathbb{Z} / 2 \mathbb{Z} \imath \mathbb{Z}$ show that $r g(n)$ may have arbitrary fast decay, just because the group has a subgroup of index 2 isomorphic to itself, so iterating this fact one gets a descending sequence of groups of growing index power of 2 but a fixed rank $=2$ (see for instance 4]).

We suggest the following definition. Let $f(n), g(n)$ be two increasing functions of natural argument $n$ taking values in $\mathbb{N}$ and having the limit $\infty$ when $n \rightarrow \infty$. 


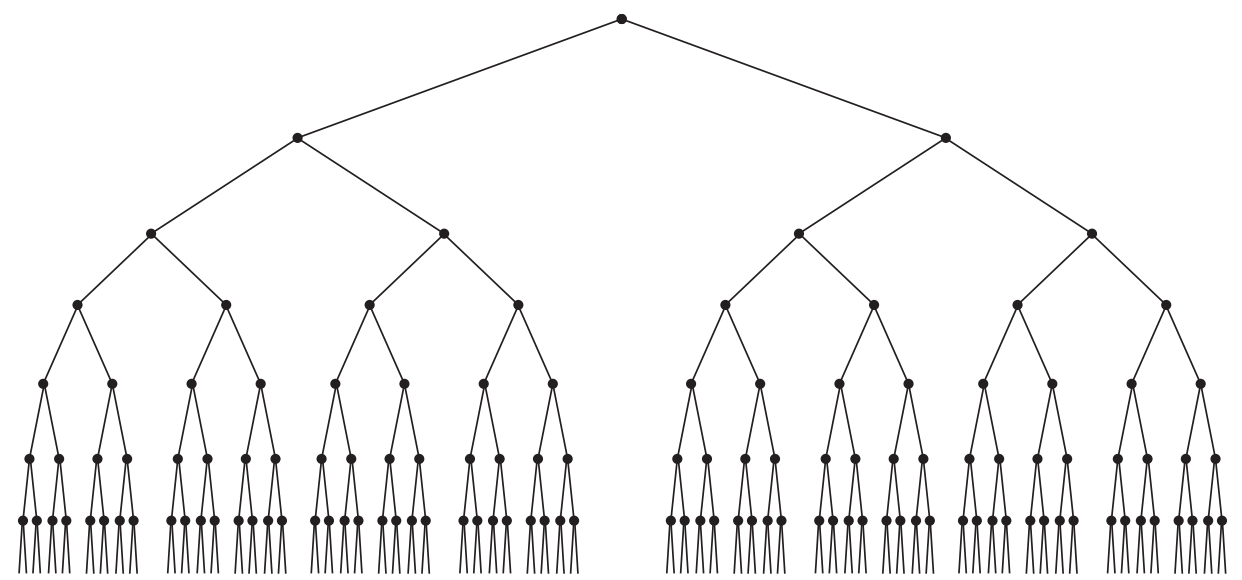

Figure 1: Binary Tree

Definition 1.1. (a) A finitely generated group $G$ is $(f, g)-R G$-rigid if there is $C \in \mathbb{N}$ such that for every subgroup $H<G$ of finite index

$$
g(d(H))<C f(C[G: H]) \text { and } f([G: H])<C g(C d(H)) .
$$

(b) $G$ is normally $(f, g)-R G$-rigid if previous inequalities hold for each normal subgroup $H \triangleleft G$ of finite index.

For instance the free group $F_{r}$ of rank $r \geq 1$ is $(f, g)-R G$-rigid where $f(n)=g(n)=n$ as in this case the ratio $(d(H)-1) /\left(\left[F_{r}: H\right]\right)$ is constant and equal to $r-1$. Also $(n, n)-R G$-rigid are all groups with $R G(G)>0$. For finitely presented groups this hold if and only if $G$ is "large" in the sense of S. Pride, i.e. contains a subgroup of finite index that surjects onto a noncommutative free group, as shown in 12.

We present the following two results. Let $\mathcal{G}=\langle a, b, c, d\rangle$ be an infinite 2 -group constructed by the first author in [10. Recall that it has intermediate growth between polynomial and exponential and has many other interesting properties [5, 6, 9, $\mathcal{G}$ has many other ways to be defined but for us it will be important that it has a natural action by automorphisms

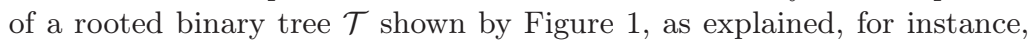
in [6].

Theorem 1.2. The group $\mathcal{G}$ is normally $(f, g)-R G$-rigid with

$$
f(n)=\log \log (n), g(n)=\log (n),
$$

Moreover, there there is a constant $D>1$ such that

$$
\frac{1}{D} \log (d(H)) \leq \log \log ([\mathcal{G}: H]) \leq D \log (d(H))
$$

hold for every nontrivial normal subgroup $H \triangleleft \mathcal{G}$. 
Conjecture 1.3. The group $\mathcal{G}$ is $(f, g)-R G$-rigid with the same functions $f, g$ as in previous theorem.

If the conjecture is true then we have an interesting rigidity property concerning the rank and index of finite index subgroups. At least we have this property for normal subgroups as shows the above theorem. Recall that all normal subgroups in $\mathcal{G}$ have finite index because $\mathcal{G}$ is justinfinite (i.e. infinite, but every proper quotients finite) as shown in [6]. Interestingly, the group $\mathcal{G}$ was used by M. Lackenby in 12 to demonstrate some phenomenon that may hold for the rank gradient. The present article develops the observation made by $\mathrm{M}$. Lackenby concerning the rank gradient in $\mathcal{G}$.

At the moment we are able only to confirm the conjecture for important subclass of subgroups of finite index in $\mathcal{G}$, namely for stabilizers of vertices of the binary rooted tree $\mathcal{T}$ on which the group $\mathcal{G}$ acts. The vertices of $\mathcal{T}$ are in bijection with finite words over binary alphabet $\{0,1\}$. Let $v$ be a vertex and $s t_{\mathcal{G}}(v)$ be its stabilizer which has index $2^{n}$ in $\mathcal{G}$ if $v$ is a vertex of level $n$.

Theorem 1.4. There is a constant $D$ such that the inequalities (3) hold for all subgroups $H=s t_{\mathcal{G}}(v)$ where $v$ run over the set of vertices in $\mathcal{T}$. In fact

$$
\frac{d(H)-1}{[\mathcal{G}: H]}=\frac{n+3}{2^{n}}
$$

if $v$ is a vertex of level $n \geq 2$.

Observe that this result is announced in [4].

\section{The group $\mathcal{G}$}

We recall some basic facts about the group $\mathcal{G}$ and its subgroups. $\mathcal{G}$ can be defined as a group of automorphisms of a rooted binary tree $\mathcal{T}$ shown in Figure 1 (the root, corresponding to the empty word, is a fixed point for the action). The generators $a, b, c, d$ of $\mathcal{G}$ are involutions, the elements $b, c, d$ commute and together with the identity element they constitute the Klein group $\mathbb{Z}_{2} \times \mathbb{Z}_{2}$. The stabilizer of the first level $H=s t_{\mathcal{G}}(1)$ is a subgroup of index 2 in $\mathcal{G}$ generated by elements $b, c, d, b^{a}, c^{a}, d^{a}$ (where $\left.x^{y}=x^{-1} y x\right)$, and the restrictions of $H$ on the left and right subtrees $\mathcal{T}_{0}, \mathcal{T}_{1}$ with the roots at vertices 0,1 determine surjective homomorphisms $\varphi_{0}, \varphi_{1}: H \rightarrow \mathcal{G}$. The direct product $\psi=\varphi_{0} \times \varphi_{1}$ of them determines the embedding $H \rightarrow \mathcal{G} \times \mathcal{G}$ and acts on generators as:

$$
b \rightarrow(a, c), c \rightarrow(a, d), d \rightarrow(1, b), b^{a} \rightarrow(c, a), c^{a} \rightarrow(d, a), d^{a} \rightarrow(b, 1)
$$

Together with the information that the generator $a$ permute the subtrees $\mathcal{T}_{0}, \mathcal{T}_{1}$ (without extra action inside them), this uniquely determines the group $\mathcal{G}$. 
The action of $\mathcal{G}$ on $\mathcal{T}$ also can be described by the following recursive rules:

$$
\begin{array}{ll}
a(0 w)=1 w, & a(1 w)=0 w \\
b(0 w)=0 a(w), & b(1 w)=1 c(w), \\
c(0 w)=0 a(w), & c(1 w)=1 d(w), \\
d(0 w)=0 w, & d(1 w)=1 b(w),
\end{array}
$$

where $w \in\{0,1\}^{*}$, and $\{0,1\}^{*}$ denotes the set of all finite words over the binary alphabet. The important property of the action of $\mathcal{G}$ on $\mathcal{T}$ is level transitivity, i.e. transitivity of the action on each level $V_{n}=\{0,1\}^{n}$.

Additionally to the stabilizers $s t_{\mathcal{G}}(n)$ of levels $n=1,2, \ldots$, an important descending series of normal subgroups is the series of rigid stabilizers rist $_{\mathcal{G}}(n)$ which are subgroups generated by rigid stabilizers rist $_{\mathcal{G}}(v), v \in$ $\{0,1\}^{n}$ of vertices of the $n$th level, and rist $_{\mathcal{G}}(v)$ is a subgroup in $\mathcal{G}$ fixing vertex $v$ and consisting of elements acting trivially outside the subtree $\mathcal{T}_{v}$ in $\mathcal{T}$ with a root at $v$. The rigid stabilizers of distinct vertices of the same level commute and are conjugate (because of the level transitivity). Thus algebraically the $\operatorname{rist}_{\mathcal{G}}(n)$ is a direct product of copies of the same group (which may depend on the level $n$ in general case). Observe that $\left\{s t_{\mathcal{G}}(n)\right\}_{n=1}^{\infty}$ and $\left\{\text { rist }_{\mathcal{G}}(n)\right\}_{n=1}^{\infty}$ are descending chains of normal subgroups of finite index with trivial intersection. The structure of groups $\operatorname{st}_{\mathcal{G}}(n)$ and $\operatorname{rist}_{\mathcal{G}}(n)$ is well understood and described in 2 .

Let $B=\langle b\rangle^{\mathcal{G}}$ be a normal closure of generator $b$ and $K=\left\langle(a b)^{2}\right\rangle^{\mathcal{G}}$. For each $n$ there is a natural embedding $\psi_{n}: s t_{\mathcal{G}}(n) \rightarrow \mathcal{G} \times \cdots \times \mathcal{G}$ into a direct product of $2^{n}$ copies of $\mathcal{G}$ which is the $n$th iteration of the embedding $\psi$ and has a geometric meaning of the attaching to the element $g \in s_{\mathcal{G}}(n)$ the $2^{n}$-tuple $\left(g_{1}, \ldots, g_{2^{n}}\right)$ of its restrictions on the subtrees with the roots at the $n$th level. Instead writing

$$
\psi_{n}(g)=\left(g_{1}, \ldots, g_{2^{n}}\right)
$$

we will write

$$
g=\left(g_{1}, \ldots, g_{2^{n}}\right)
$$

In particular the relations (4) can be rewritten as $b=(a, c), c=(a, d), d=$ $(1, b), b^{a}=(c, a), c^{a}=(d, a), d^{a}=(b, 1)$.

The important facts about groups $s t_{\mathcal{G}}(n)$ and rist $_{\mathcal{G}}(n)$ are that the $\psi_{n-3}$ image of $s t_{\mathcal{G}}(n)$ has the decomposition

$$
s t_{\mathcal{G}}(3) \times s t_{\mathcal{G}}(3) \times \cdots \times s t_{\mathcal{G}}(3)
$$

(products of $2^{n-3}$ copies of $\left.s t_{\mathcal{G}}(3)\right)$ when $n \geq 4$, and the $\psi_{n}$ image of rist $_{\mathcal{G}}(n)$ has the decomposition

$$
K \times K \times \cdots \times K
$$

(product of $2^{n}$ copies of $K$ ) when $n \geq 2$ (see 2]). We will use later the notations $K_{n}$ for rist $_{\mathcal{G}}(n)$ when $n \geq 2$ and keep in mind the decompositions (5) and (6). We also denote by $K_{1}$ a subgroup in $s t_{\mathcal{G}}(1)$ whose $\psi$-image is $K \times K$. The group $\mathcal{G}$ is regularly branched over $K$ as $K_{1}$ is a subgroup of $K$, and $\left[\mathcal{G}: K_{1}\right]<\infty$. Thus $\mathcal{G}$ contains subgroups shown by Figure 2. To each level $n$ corresponds a group $K_{n}$ that fixes each vertex $v$ of this level and whose restriction to the subtree $\mathcal{T}_{v}$ with the root $v$ is $K$ (if to identify $\mathcal{T}_{v}$ with $\mathcal{T}$ ). Moreover $K_{n}$ is a direct product of these projections. 


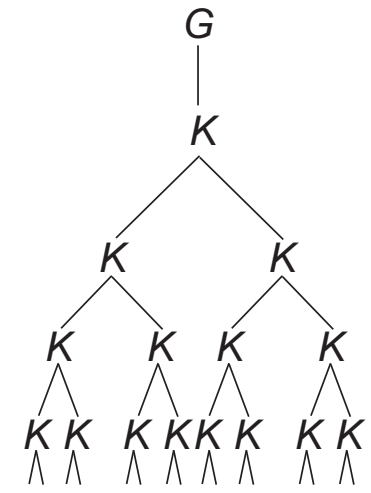

Figure 2: Structure of branching subgroups

\section{Proof of Theorem 1.2}

We begin this section with reminding that the group $\mathcal{G}$ is branch group as defined in [6, because it acts level transitive on the tree $\mathcal{T}$ and rigid stabilizers rist $_{\mathcal{G}}(n), n=1,2, \ldots$ have finite index in $\mathcal{G}$. The branch structure of $\mathcal{G}$ that we will use is given by the Figure 2 and was mentioned in previous section. The proof of the theorem 1.2 is based on the following

Proposition 3.1. Let $N \triangleleft \mathcal{G}$ be a nontrivial normal subgroup. Let $n$ be a smallest nonnegative integer such that $N<s t_{\mathcal{G}}(n)$ but $N$ is not a subgroup of $s t_{\mathcal{G}}(n+1)$. Then

(a)

$$
s t_{\mathcal{G}}(n+6)<N
$$

(b) If $n \geq 4$ then

$$
\operatorname{rist}_{\mathcal{G}}(n+3)<N<\operatorname{rist}_{\mathcal{G}}(n-3)
$$

Proof. For the part (a) we first address the reader to the proof of the theorem 4 given in [6] as we will follow the same line in our arguments. First, let us make a comparison of notations used in [6] and here. The branch structure for the group $\mathcal{G}$ is given in our case by the pair $\left(\left\{L_{n}\right\},\left\{H_{n}\right\}\right)$ where $L_{n}=K$ and $H_{n}=K_{n}$ for all $n$. The reader should keep in mind the picture given by the figure 2

Also, the normal subgroup in the statement of theorem 4 is denoted by $P$ while in the proposition under consideration it is denoted $N$. The proof of theorem 4 from [6] (modulo of the change of notations) shows that if $N<s t_{\mathcal{G}}(n)$ but $N$ is not a subgroup of $s t_{\mathcal{G}}(n+1)$, then $N$ contains commutator subgroup $K_{n+1}^{\prime}=\left[K_{n+1}, K_{n+1}\right]$ (recall that rist $t_{\mathcal{G}}(n)=K_{n}$ if $n>1$ ). By proposition 9 from [6] $K^{\prime}=K_{2}$, hence $K_{n+1}^{\prime}=K \times K \times \cdots \times K$ $\left(2^{n+3}\right.$ factors $)$ so $K_{n+1}^{\prime}=K_{n+3}$, and $N>K_{n+3}$, which gives the lower inclusion in part (b).

Also by proposition 9 from [6] we have the inclusion $K>s t_{\mathcal{G}}(3)$ from which, together with the fact presented by factorization (5) we conclude 
that $K_{n+3}>s t_{\mathcal{G}}(n+6)$ and thus $N>s t_{\mathcal{G}}(n+6)$. The part (a) is thus established. To get the upper inclusion in part (b) under assumption that $n>3$ we observe that the inclusion $K>s t_{\mathcal{G}}(3)$ and factorization (5) imply that $K_{n-3}>s t_{\mathcal{G}}(n)$, therefore we are done.

The group $\mathcal{G}$ is not virtually cyclic (by many reasons, for instance because it is finitely generated infinite torsion group). Let $N \triangleleft G$ be a nontrivial normal subgroup. It is automatically of finite index, as $\mathcal{G}$ is justinfinite group 6] (i.e. infinite group with every proper quotient finite). In fact we can assume from the beginning that $N$ is a normal subgroup of finite index when proving theorem 1.2 (so the just-infiniteness property is not needed). If $n$ in the statement of proposition 3.1 is less than 4, then there are only finitely many subgroups in $\mathcal{G}$ containing $s t_{\mathcal{G}}(5)$, their ranks are $\geq 2$, and so there is a constant $D$ satisfying the condition of the theorem 1.2 Therefore we can assume that $n \geq 4$. Now apply part (b) of the proposition.

The quotient rist $\mathcal{G}(n-3) /$ rist $_{\mathcal{G}}(n+3)=K_{n-3} / K_{n+3}$ is isomorphic to

$$
A:=\left(K / K_{6}\right)^{2^{n-3}}=K / K_{6} \times \cdots \times K / K_{6},
$$

( $2^{n-3}$ factors). The group $K / K_{6}$ is a finite 2-group of certain nilpotency class $l$ (for us it is not important the exact value of $l$ ). Therefore $A$ is nilpotent of the class $l$ as well and it is generated by not more than $3 \cdot 2^{n-3}$ elements as $K$ is 3 -generated group [6]. It is well known that a subgroup of finitely generated nilpotent group is finitely generated and there is a universal upper bound on the ranks of subgroups of nilpotent group $G$ in terms of $d(G)$ and class of nilpotency $c$ of $G$.

We use the most simple upper bound given by the following lemma

Lemma 3.2. Let $G$ be a finitely generated nilpotent group of class $c$. Then for every subgroup $H<G$ the upper bound

$$
d(H)<d(G)^{c}
$$

holds.

Proof. If $c=1$ then $G$ is abelian and thus $d(H) \leq d(G)$. For $G$ of class $c$ let $\gamma_{1}(G)=G$ and $\gamma_{i+1}(G)=\left[G, \gamma_{i}(G)\right] i=1,2, \ldots$ be the elements of the lower central series. Suppose $G$ is generated by set $S$. Each factor $\gamma_{i}(G) / \gamma_{i+1}(G)$ is generated by the iterated commutators $\left[s_{1},\left[s_{2}, \ldots\right]\right.$ of length $i+1$, where $s_{j} \in S$. Thus $d\left(\gamma_{i}(G) / \gamma_{i+1}(G)\right) \leq d(G)^{i+1}$. Denote $H_{i}=H \cap \gamma_{i}(G)$. Then $H_{i} \gamma_{i+1}(G) / \gamma_{i+1}(G)$ is an abelian group, and thus $d\left(H_{i} \gamma_{i+1}(G) / \gamma_{i+1}(G)\right) \leq d\left(\gamma_{i}(G) / \gamma_{i+1}(G)\right) \leq d(G)^{i+1}$. Note that

$$
d(H) \leq d\left(H \gamma_{1}(G) / \gamma_{1}(G)\right)+d\left(H \cap \gamma_{1}(G)\right) \leq d(G)+d\left(H_{1}\right) .
$$

Applying this iteratively we obtain

$$
\begin{aligned}
& d(H) \leq d(G)+d\left(H_{1}\right) \leq d(G)+d\left(H_{1} \gamma_{2}(G) / \gamma_{2}(G)\right)+d\left(H_{2}\right) \leq \\
& d(G)+d\left(\gamma_{1}(G) / \gamma_{2}(G)\right)+d\left(H_{2}\right) \leq \\
& d(G)+d(G)^{2}+d\left(H_{2}\right) \leq \cdots \leq d(G)+\cdots+d(G)^{c-1} \leq d(G)^{c}
\end{aligned}
$$


Using this lemma we get

$$
d(N)<\left(3 \cdot 2^{n-3}\right)^{l+2}=3^{l+2} 2^{(n-3)(l+2)}<a_{1} 2^{a_{2} n}
$$

for some positive constants $a_{1}, a_{2}$.

Now we are going to give a lower bound for $d(N)$. We factorize the inclusions $K_{n+3}<N<K_{n-3}$ by $K_{n+3}^{\prime}$ getting

$$
K_{n+3} / K_{n+3}^{\prime}<N / K_{n+3}^{\prime}<K_{n-3} / K_{n+3}^{\prime}
$$

The group $K_{n-3} / K_{n+3}^{\prime}$ is a direct product of $2^{n-3}$ copies of the group $K / K_{8}$ as $K=K_{2} . K / K_{8}$ is a finite 2-group. Let $s$ be its nilpotency class, so $K_{n-3} / K_{n+3}^{\prime}$ also has nilpotency class $s$. As $\bar{N}:=N / K_{n+3}^{\prime}$ is a subgroup of $K_{n-3} / K_{n+3}^{\prime}$ its class of nilpotency is $\leq s$.

The group $K_{n+3} / K_{n+3}^{\prime}$ is a direct product of $2^{n+3}$ copies of the group $K / K^{\prime}=K / K_{2}$. Let $t=d\left(K / K^{\prime}\right)($ in fact $t=3)$. Then $d\left(K_{n+3} / K_{n+3}^{\prime}\right)=$ $t 2^{n+3}$. Using lemma 3.2 we conclude

$$
d\left(K_{n+3} / K_{n+3}^{\prime}\right)=t 2^{n+3} \leq(d(N))^{s+2}
$$

from which we conclude that there are positive constants $a_{3}, a_{4}$ such that

$$
a_{3} 2^{a_{4} n} \leq d(N)
$$

Now using the part (a) of the Proposition 3.1 we provide upper and lower bounds for the index $[\mathcal{G}: N]$. For this purpose we use the fact that

$$
\left[\mathcal{G}: s t_{\mathcal{G}}(n)\right]=2^{5 \cdot 2^{n-3}+2}
$$

as shown at the end of the proof of theorem 14 from 6]. Hence part (a) of the proposition lead us to the existence of positive constants $a_{5}, a_{6}$ and constants $a_{7}, a_{8}$ such that

$$
2^{a_{5} 2^{n}+a_{7}} \leq[\mathcal{G}: N] \leq 2^{a_{6} 2^{n}+a_{8}}
$$

Taking the double logarithm with base 2 of this inequalities and applying the same logarithm to the previously obtained inequalities

$$
a_{3} 2^{a_{4} n} \leq d(N) \leq a_{1} 2^{a_{2} n}
$$

a simple calculus finishes the proof of the theorem.

\section{Proof of Theorem 1.4}

We begin the proof of Theorem 1.4. The groups $R_{n}, Q_{n}$ and $P_{n}$ which are defined below are the finite index analogs of groups $R, Q, P$ introduced and studied in 2. The recursive relations between them are analogous to the corresponding relations between $R, Q, P$ given in 2] by Theorems 4.4 and 4.5. The proof is based on a number of computations that we split in propositions and lemmas.

Let us introduce the elements $t=(a b)^{2}, u=(\text { bada })^{2}=(t, 1), v=$ $(a b a d)^{2}=(1, t)$. The direct computation gives the result of their conjugation by generators, together with the conjugation of $(a c)^{4}$, as shown in the next subsections: 


\subsection{Conjugates of $t, u, v$}

$$
\begin{aligned}
& t^{a}=t^{-1} \\
& t^{b}=t^{-1} \\
& t^{c}=t^{-1} v \\
& t^{d}=v^{-1} t \\
& t^{d d^{a}}=v^{-1} t u \\
& u^{a}=v \\
& u^{b}=u^{-1} \\
& u^{c}=u^{-1} \\
& u^{d}=u \\
& u^{d d^{a}}=u^{-1} \\
& v^{a}=u \\
& v^{b}=t^{-1} v^{-1} t \\
& v^{c}=t^{-1} v t \\
& v^{d}=v^{-1} \\
& v^{d d^{a}}=v^{-1}
\end{aligned}
$$

\subsection{Conjugates of $(a c)^{4}$}

Denote $x_{0}=(a c)^{4}$

$$
\begin{aligned}
& x_{0}^{a}=x_{0} \\
& x_{0}^{b}=(1, u) x_{0} \\
& x_{0}^{c}=x_{0} \\
& x_{0}^{d}=(1, u) x_{0} \\
& x_{0}^{d d^{a}}=(u, u) x_{0} .
\end{aligned}
$$

Now let us introduce more elements and show the result of their conjugation.

\subsection{Definition and conjugates of $x_{m}, u_{m}, v_{m}$}

Recall that rist $_{\mathcal{G}}\left(1^{m}\right)$ is the subgroup of $\mathcal{G}$ that fixes the vertex $1^{m}$ together with all vertices that do not start with $1^{m}$. It is easy to check that $(a c)^{4}, u$ and $v$ belong to $K$, and that $\psi(K) \supset K \times K$. It follows that there are such $x_{m}, u_{m}$ and $v_{m}$ in rist $\operatorname{G}_{\mathcal{G}}\left(1^{m}\right)$ that $x_{m}\left(1^{m} w\right)=1^{m}(a c)^{4}(w)$, $u_{m}\left(1^{m} w\right)=1^{m} u(w)$ and $v_{m}\left(1^{m} w\right)=1^{m} v(w)$. Note that $x_{0}=(a c)^{4}$. We compute their conjugates below (the proof is by induction, since $x_{m}=\left(1, x_{m-1}\right), u_{m}=\left(1, u_{m-1}\right)$ and $\left.v_{m}=\left(1, v_{m-1}\right)\right)$ :

$$
x_{m}^{b}= \begin{cases}u_{m+1} x_{m} & \text { if } 3 \mid m \\ x_{m} & \text { if } 3 \mid(m-1) \\ u_{m+1} x_{m} & \text { if } 3 \mid(m-2)\end{cases}
$$




$$
\begin{aligned}
& x_{m}^{c}= \begin{cases}x_{m} & \text { if } 3 \mid m \\
u_{m+1} x_{m} & \text { if } 3 \mid(m-1) \\
u_{m+1} x_{m} & \text { if } 3 \mid(m-2)\end{cases} \\
& x_{m}^{d}= \begin{cases}u_{m+1} x_{m} & \text { if } 3 \mid m \\
u_{m+1} x_{m} & \text { if } 3 \mid(m-1) \\
x_{m} & \text { if } 3 \mid(m-2)\end{cases}
\end{aligned}
$$

We have that

$$
x_{0}^{d d^{a}}=(u, u) x_{0},
$$

and for $m>0$

$$
\begin{aligned}
& x_{m}^{d d^{a}}=\left(1, x_{m-1}^{b}\right)= \begin{cases}u_{m+1} x_{m} & \text { if } 3 \mid m \\
u_{m+1} x_{m} & \text { if } 3 \mid(m-1) \\
x_{m} & \text { if } 3 \mid(m-2)\end{cases} \\
& x_{1}^{x_{0}}=\left(1, x_{0}^{d d^{a}}\right)=(1,1, u, u)\left(1, x_{0}\right)=(1,1, u, u) x_{1}
\end{aligned}
$$

When $n+1<m$ we have,

$$
x_{m}^{x_{n}}= \begin{cases}x_{m} & \text { if } 3 \mid(m-n) \\ u_{m+1} x_{m} & \text { if } 3 \chi(m-n)\end{cases}
$$

Here is the list of conjugates of $u_{m}$ and $v_{m}$ :

$$
\begin{gathered}
u_{m}^{b}= \begin{cases}u_{m}^{-1} & \text { if } 3 \mid m \\
u_{m}^{-1} & \text { if } 3 \mid(m-1) \\
u_{m} & \text { if } 3 \mid(m-2)\end{cases} \\
u_{m}^{d d^{a}}= \begin{cases}u_{m} & \text { if } 3 \mid m \\
u_{m}^{-1} & \text { if } 3 \mid(m-1) \\
u_{m}^{-1} & \text { if } 3 \mid(m-2)\end{cases} \\
v_{m}^{b}= \begin{cases}v_{m-1}^{-1} v_{m}^{-1} v_{m-1} & \text { if } 3 \mid m \\
v_{m-1}^{-1} v_{m} v_{m-1} & \text { if } 3 \mid(m-1) \\
v_{m}^{-1} & \text { if } 3 \mid(m-2)\end{cases} \\
v_{m}^{d d^{a}}= \begin{cases}v_{m}^{-1} & \text { if } 3 \mid m \\
v_{m-1}^{-1} v_{m}^{-1} v_{m-1} & \text { if } 3 \mid(m-1) \\
v_{m-1}^{-1} v_{m} v_{m-1} & \text { if } 3 \mid(m-2)\end{cases}
\end{gathered}
$$

In the next two subsections we introduce sequences of subgroups $R_{n}$ and $Q_{n}, n=1,2, \ldots$ and prove some structural results about them.

\subsection{Groups $R_{n}$}

Let $R_{1}=K=\langle t, u, v\rangle$. Let $R_{n}=\left(K \times R_{n-1}\right)\left\{1,(a c)^{4}\right\}$ for $n \geq 2$. Then Proposition 4.1.

$$
\begin{gathered}
R_{2}=\left\langle x_{0}, u_{0}, u_{1}, v_{0},(u, u)\right\rangle \\
R_{n}=\left\langle x_{0}, \ldots, x_{n-2}, u_{0}, u_{1}, u_{2}, v_{n-2},(u, u)\right\rangle
\end{gathered}
$$

for $n \geq 3$. 
Proof. Since $R_{2}=(K \times K)\left\{1, x_{0}\right\}$, it follows that

$$
R_{2}=\left\langle x_{0},(t, 1),(u, 1),(v, 1),(1, t),(1, u),(1, v)\right\rangle .
$$

Now, by (77) $t^{d d^{a}}=v^{-1} t u$, and since $x_{0}=\left(d d^{a}, d d^{a}\right)$, we have that $(t, 1)^{x_{0}}=(v, 1)^{-1}(t, 1)(u, 1)$, and the same for $(1, t)$. So we can discard $(v, 1),(1, v)$ from the list. Notice also that multiplying $(u, 1)$ by $(1, u)$ we can replace $(u, 1)$ with $(u, u)$. Since $u_{0}=u=(t, 1), u_{1}=(1, u)$, and $v_{0}=v=(1, t)$, we obtain the generators for $R_{2}$.

We have $R_{3}=\left(K \times R_{2}\right)\left\{1, x_{0}\right\}$, hence $R_{3}$ is generated by

$$
R_{3}=\left\langle x_{0},(t, 1),(u, 1),(v, 1), x_{1}, u_{1}, u_{2}, v_{1},(1,1, u, u)\right\rangle .
$$

Where $x_{1}=\left(1, x_{0}\right), u_{1}=\left(1, u_{0}\right), u_{2}=\left(1, u_{1}\right)$ and $v_{1}=\left(1, v_{0}\right)$.

In the same way as for $R_{2}$ we can discard $(v, 1)$ and replace $(u, 1)$ by $(u, u)$. By (14) $x_{1}^{x_{0}}=(1,1, u, u) x_{1}$, and so we can discard $(1,1, u, u)$, and we are done.

Now suppose

$$
R_{n}=\left\langle x_{0}, \ldots, x_{n-2}, u_{0}, u_{1}, u_{2}, v_{n-2},(u, u)\right\rangle
$$

Then from the formula $R_{n+1}=\left(K \times R_{n}\right)\left\{1,(a c)^{4}\right\}$ we obtain that $R_{n+1}$ is generated by

$$
\left\langle x_{0},(t, 1),(u, 1),(v, 1), x_{1}, \ldots, x_{n-1}, u_{1}, u_{2}, u_{3}, v_{n-1},(1,1, u, u)\right\rangle .
$$

As above, we can discard $(v, 1)$ and $(1,1, u, u)$ and replace $(u, 1)$ with $(u, u)$. It is left to note that $x_{2}^{x_{0}}=u_{3} x_{2}$ by (15), so $u_{3}=\left[x_{0}, x_{2}\right]$ and hence we can discard it from the list.

For any group $G$ define $G^{(2)}=G / G\left(X^{2}\right)$, where $G\left(X^{2}\right)$ is the subgroup generated by all squares of elements in $G$. Then $G^{(2)}$ is elementary abelian 2 -group, and so $\operatorname{dim}_{\mathbb{F}_{2}} G^{(2)}$ is defined.

Proposition 4.2. $\operatorname{dim}_{\mathbb{F}_{2}} R_{n}^{(2)}=n+4$ for $n \geq 3, \operatorname{dim}_{\mathbb{F}_{2}} R_{2}^{(2)}=5$, and $\operatorname{dim}_{\mathbb{F}_{2}} K^{(2)}=3$.

Proof. The equality for $K$ follows from the theory of the group $\mathcal{G}$, see [2, 6, 9].

To prove the rest, we need the following obvious lemma

Lemma 4.3. Suppose $G=H \rtimes(\mathbb{Z} / 2 \mathbb{Z})$. Let $\alpha: H^{(2)} \rightarrow H^{(2)}$ be the operator on $H^{(2)}$ induced by the action of $\mathbb{Z} / 2 \mathbb{Z}$. Then $G^{(2)}=H^{(2)} /(1+$ $\alpha)\left(H^{(2)}\right) \oplus \mathbb{Z} / 2 \mathbb{Z}$.

Note that by (7), (8), (9), $d d^{a}$ induces the following action on $K^{(2)}$ : $[t, u, v] \mapsto[t+u+v, u, v]$. Hence $\left(1+d d^{a}\right)\left(K^{(2)}\right)=\{0, u+v\}$. Thus $\operatorname{dim}_{\mathbb{F}_{2}} R_{2}^{(2)}=2+2+1=5$.

Now, it follows from (12), (13) that $\left(1+d d^{a}\right)\left(x_{0}\right)=(u, u),(1+$ $\left.d d^{a}\right)\left(x_{1}\right)=u_{2}$ and $\left(1+d d^{a}\right)\left(x_{m}\right)=0$ for $m>1$, since from (15) we have for $m \geq 3$ that $u_{m}=\left[x_{m-3}, x_{m-1}\right]$.

Also, from (17), (18), $\left(1+d d^{a}\right)\left(u_{m}\right)=0,\left(1+d d^{a}\right)\left(v_{m}\right)=0$ for all $m \geq 0$, and $\left(1+d d^{a}\right)((u, u))=0$.

Thus $\left(1+d d^{a}\right)\left(R_{2}^{(2)}\right)=\{0,(u, u)\}$, and so $\operatorname{dim}_{\mathbb{F}_{2}} R_{3}^{(2)}=2+5-1+1=7$. Analogously, $\left(1+d d^{a}\right)\left(R_{n}^{(2)}\right)=\left\langle(u, u), u_{2}\right\rangle$ for $n \geq 3$, and so by induction $\operatorname{dim}_{\mathbb{F}_{2}} R_{n+1}^{(2)}=2+\operatorname{dim}_{\mathbb{F}_{2}} R_{n}^{(2)}-2+1=n+5$. 
Corollary 4.4. $d\left(R_{1}\right)=3, d\left(R_{2}\right)=5, d\left(R_{n}\right)=n+4$ for $n \geq 3$.

\subsection{Groups $Q_{n}$}

Let $Q_{1}=B=\langle b, t, u, v\rangle=K \rtimes\{1, b\}$ and $Q_{n}=\left(K \times R_{n-1}\right)\left\langle b,(a c)^{4}\right\rangle$ for $n>1$.

Proposition 4.5. $Q_{n}=R_{n} \rtimes\{1, b\}$.

$$
\begin{gathered}
Q_{2}=\left\langle b, x_{0}, u_{0}, v_{0},(u, u)\right\rangle . \\
Q_{n}=\left\langle b, x_{0}, x_{1}, \ldots, x_{n-2}, u_{0}, u_{2}, v_{n-2},(u, u)\right\rangle .
\end{gathered}
$$

for $n \geq 3$.

Proof. The proof is analogous to the proof of Proposition 4.1 using lists of generators for $R_{n}$ from the statement of Proposition 4.1 and the additional fact that $x_{0}^{b}=u_{1} x_{0}$, hence $u_{1}=x_{0}^{b} x_{0}$.

Proposition 4.6. $\operatorname{dim}_{\mathbb{F}_{2}} Q_{n}^{(2)}=n+4$ for $n \geq 3, \operatorname{dim}_{\mathbb{F}_{2}} Q_{2}^{(2)}=5$, and $\operatorname{dim}_{\mathbb{F}_{2}} Q_{1}^{(2)}=4$.

Proof. Note that to compute $\operatorname{dim}_{\mathbb{F}_{2}} Q_{n}^{(2)}$ we may use the Lemma4.3 since $Q_{n}=R_{n} \rtimes\{1, b\}$. Thus we need to compute the induced action of $b$ on $R_{n}^{(2)}$.

Note that $(1+b)\left(K^{(2)}\right)=0$, by (7), (8), (9). Also, $(1+b)\left(x_{0}\right)=$ $u_{1},(1+b)\left(x_{m}\right)=0$ for $m \geq 1$ by (10). $(1+b)\left(u_{m}\right)=0$ for $m \geq$ 0 by (16). Finally, $(1+b)((u, u))=(u, u)+(v, 1)+(1, u)$, and since $(t, 1)^{x_{0}}=(v, 1)^{-1}(t, 1)(u, 1)$, it follows that $(v, 1)+(u, 1)=0$, and thus $(1+b)((u, u))=(u, u)+(v, 1)+(1, u)=0$.

Hence $(1+b)\left(R_{n}^{(2)}\right)=\left\langle u_{1}\right\rangle$, and thus $\operatorname{dim}_{\mathbb{F}_{2}} Q_{2}^{(2)}=5-1+1=5$, and $\operatorname{dim}_{\mathbb{F}_{2}} Q_{n}^{(2)}=n+4-1+1=n+4$.

Corollary 4.7. $d\left(Q_{1}\right)=4, d\left(Q_{2}\right)=5, d\left(Q_{n}\right)=n+4$ for $n \geq 3$.

Finally we introduce groups $P_{n}$.

\subsection{Groups $P_{n}$}

Let $P_{n}=s t_{\mathcal{G}}\left(1^{n}\right)$. Then $P_{1}=s t_{\mathcal{G}}(1)$, and $P_{n}=\left(K \times Q_{n-1}\right)\left\langle c,(a c)^{4}\right\rangle$ for $n>1$.

Proposition 4.8. $P_{n}=R_{n} \rtimes\{1, b, c, d\}$ and

$$
P_{n}=\left\langle c, d, x_{0}, x_{1}, \ldots, x_{n-2}, u_{0}, v_{n-2},(u, u)\right\rangle,
$$

for $n \geq 2$.

Proof. Analogous to the proofs of Proposition 4.1 and Proposition 4.5 using the additional fact that $x_{1}^{d}=u_{2} x_{1}$ by (11).

Proposition 4.9 .

$$
\operatorname{dim}_{\mathbb{F}_{2}} P_{n}^{(2)}=n+4
$$

for $n \geq 2$, and $\operatorname{dim}_{\mathbb{F}_{2}} P_{1}^{(2)}=4$. 
Proof. For $n=1$ it follows from $P_{1}=s t(1)=\left\langle d, c, d^{a}, c^{a}\right\rangle$. For $n \geq 2$ it follows from $P_{n}=R_{n} \rtimes\{1, b, c, d\}$ and a slight generalization of the Lemma $4.3 P_{n}^{(2)}=\left(R_{n}^{(2)} / V_{n}\right) \oplus \mathbb{F}_{2}^{2}$, where $V_{n}=(1+b) R_{n}^{(n)}+(1+c) R_{n}^{(n)}$.

Now we are ready to prove theorem 1.4 As $\mathcal{G}$ acts level transitive on $\mathcal{T}$, for each vertex $v$ of the level $n$ the group $s t_{\mathcal{G}}(v)$ is conjugate in $\mathcal{G}$ to $P_{n}$. From Proposition 4.8 it follows that the minimum number of generators of $P_{n}$ is $\leq n+4$. The last proposition shows that it is exactly $n+4$ when $n \geq 2$. As index of $P_{n}$ in $\mathcal{G}$ is $2^{n}$ (because of the level transitivity of $\mathcal{G}$ ) the ratio

$$
\frac{d(H)-1}{[\mathcal{G}: H]}=\frac{n+3}{2^{n}}
$$

for $H=s t_{\mathcal{G}}(v)$ where vertex $v$ belongs to the level $n \geq 2$. If $v$ is a vertex of the first level then $H=s t_{\mathcal{G}}(1)$ is a subgroup of index 2 and is 4-generated (by elements $b, c, b^{a}, c^{a}$ ). Taking all this into account we get the conclusion of the theorem 1.4

\section{References}

[1] Miklós Abért and Nikolay Nikolov. Rank gradient, cost of groups and the rank versus Heegaard genus problem. J. Eur. Math. Soc. (JEMS), 14(5):1657-1677, 2012.

[2] Laurent Bartholdi and Rostislav I. Grigorchuk. On parabolic subgroups and Hecke algebras of some fractal groups. Serdica Math. J., 28(1):47-90, 2002.

[3] N.N. Bogolyubov. On some ergodic properties of continious groups of transformations. Nauk. Zap. Kïv Derzh. Univ. im. T.G.Shevchenka, 4(5):45-52, 1939.

[4] R. Grigorchuk and R. Kravchenko. On the lattice of subgroups of the lamplighter group. Internat. J. Algebra Comput., 24(6):837-877, 2014.

[5] R. I. Grigorchuk. Degrees of growth of finitely generated groups and the theory of invariant means. Izv. Akad. Nauk SSSR Ser. Mat., 48(5):939-985, 1984.

[6] R. I. Grigorchuk. Just infinite branch groups. In New horizons in pro-p groups, volume 184 of Progr. Math., pages 121-179. Birkhäuser Boston, Boston, MA, 2000.

[7] R. I. Grigorchuk. Some problems of the dynamics of group actions on rooted trees. Tr. Mat. Inst. Steklova, 273(Sovremennye Problemy Matematiki):72-191, 2011.

[8] R. I. Grigorchuk, V. V. Nekrashevich, and V. I. Sushchanskiŭ. Automata, dynamical systems, and groups. Tr. Mat. Inst. Steklova, 231(Din. Sist., Avtom. i Beskon. Gruppy):134-214, 2000.

[9] Rostislav Grigorchuk. Solved and unsolved problems around one group. In Infinite groups: geometric, combinatorial and dynamical aspects, volume 248 of Progr. Math., pages 117-218. Birkhäuser, Basel, 2005 . 
[10] R. I. Grigorčuk. On Burnside's problem on periodic groups. Funktsional. Anal. i Prilozhen., 14(1):53-54, 1980.

[11] F. J. Grunewald, D. Segal, and G. C. Smith. Subgroups of finite index in nilpotent groups. Invent. Math., 93(1):185-223, 1988.

[12] Marc Lackenby. Expanders, rank and graphs of groups. Israel J. Math., 146:357-370, 2005.

[13] Alexander Lubotzky and Dan Segal. Subgroup growth, volume 212 of Progress in Mathematics. Birkhäuser Verlag, Basel, 2003.

[14] John von Neumann. Zurr allgemeinen theorie des masses. Fund.Math., 13:73-116, 1929. 